\title{
Exciting news for the 2021 advances in computer games conference
}

This year's Advances in Computer Games conference (ACG 2021) will be held online over November 23-25. The conference co-chairs are Cameron Browne (University of Maastricht) and Akihiro Kishimoto (IBM Research, Tokyo). See https://icga.org/?page_id=3086 for the latest details.

We are delighted to make two special announcements regarding the conference:

1. It's free! There will be no registration fee for anyone wanting to watch any of the conference talks. We've never done this before and hope that this will help popularize the ICGA and our activities. Note that those presenting a paper may still pay a small conference registration fee.

2. Fantastic keynote speakers! We are delighted to announce that there will be three keynote talks:

- David Silver (DeepMind, London). David was the lead architect on the breakthrough ALPHAGo program (and its successors).

- Michael Bowling (DeepMind, Edmonton and the University of Alberta). Michael is the lead of the University of Alberta's Computer Poker Research Group. In 2015 his team announced that they had solved two-player poker, and followed that up with a superhuman multi-player poker program.

- Mark Lefler (Komodo Dragon) and Larry Kaufman (Komodo Dragon). Mark is well known for his work on computer chess programs and the impressive ZILLIONS OF GAMES. Larry has been a major force behind the scenes for many chess programs over many decades.

Please join us for ACG 2021. Details will be posted on the ICGA web site (https://icga.org). 